\title{
Biofilm formation by Candida albicans and Candida glabrata isolated from urine specimens of diabetic Iraqi women
}

\author{
Enas Abdullah Ibrahim, Rafea Qasim Hamzah* \\ Department of Biology, College of Education for Pure Science, University of Mosul, Iraq \\ (enas34ibrahim@gmail.com)(dr.rafeaqm@uomosul.edu.iq) \\ *Correspondence:dr.rafeaqm@uomosul.edu.iq
}

\begin{abstract}
Biofilms are multicellular communities where microorganisms are grown and form an extracellular matrix that protects the pathogenic microorganisms from the immunity system and antimicrobial agents. This study is aimed to identify Candida spp. isolated from urine specimens by using traditional techniques, germ tube, growth on corn meal agar medium and chromogenic medium then determine the ability of isolates to producing biofilm by tube method (TM) and congo red agar method (CRA). In our study urine specimens were obtained from 174 diabetic females in the period of six months at the Al-Wafa Specialized Center for Diabetes and Endocrinology, Mosul city, Iraq. Out of the total 174 specimens, yeast species were isolated from $56(32.2 \%)$ specimens. Out of the 56 isolates, 50 isolates were positive for Candida spp., especially $C$. glabrata which appeared maximum in 30 isolates $(60 \%)$ and followed by $C$. albicans 18 isolates $(36 \%)$ and $C$. krusei 2 isolates $(4 \%)$. In the TM method for biofilm detection, $C$. albicans showed 16 isolates (88.9\%) positive for biofilm formation followed by 29 isolates $(96.7 \%)$ of $C$. glabrata. Furthermore, in the CRA method, all isolates (100\%) of $C$. albicans were negative followed by 27 isolates $(90 \%)$ of $C$. glabrata, whereas only 3 isolates $(10 \%)$ of $C$. glabrata were positive. We can conclude that TM is the best conventional method and is sensitive to detect biofilm-forming yeast when compared with the CRA method.
\end{abstract}

Keywords: Biofilm, Candida albicans, Candida glabrata, Diabetic women

Received: August 15 $5^{\text {th }}, 2021 /$ Accepted: Oct. 10 $0^{\text {th }}, 2021 /$ Online: Oct. $18^{\text {th }}, 2021$.

\section{INTRODUCTION}

Urinary tract infections (UTIs) are a major health concern among both outpatients and hospitalized patients (Goyal et al., 2016). It is mainly associated with diabetic patients, because diabetes alters the normal host system, which may contribute to the development of UTI (Woldemariam et al., 2019). In recent years, urinary tract infections caused by Candida opportunistic pathogens are becoming more frequent and accounted for 10-15\% of UTIs (Goyal et al., 2016; Chandak et al., 2018; He et al., 2021).

The yeast-like fungus Candida spp. is the most common cause of a wide range of superficial infections of mucosal surfaces in humans, it colonizes the gastrointestinal and reproductive systems, particularly the female vaginal tract, as well as the skin (Alfouzan and Dhar, 2017). Immunosuppressive disorders such as diabetes, HIV, chemotherapy patients, take broad-spectrum antibiotics or steroids, and indwelling medical equipment implanted in the body such as urinary catheters are all risk factors for invasive Candida infections (Devi et al., 2019). There are several factors that may lead to candiduria in diabetic patients such as female gender and uncontrolled diabetes (Geerlings et al., 2014).

Urine samples may be positive for Candida spp. for a variety of reasons: contamination or colonization, asymptomatic infection or infection with a potential for ascending pyelonephritis, renal and disseminated candidiasis, they may contribute to significant morbidity and mortality, as well as long term hospitalization and increased costs. In most individuals with candiduria there are no traditional symptoms like dysuria, urgency or fever (Alfouzan and Dhar, 2017).

Candida albicans is the most common pathogen that causes Candida infections, identified in $50-70 \%$ of candiduria cases, and about $20 \%$ of candiduria cases are caused by $C$. glabrata (Pieralli et al., 2014; Cavalheiro and Teixeira, 2018) Non-albicans strains have become more common throughout years, C. glabrata is becoming more common in urine specimens across the world and $C$. parapsilosis is prevalent etiology for candidiasis, especially neonatal candiduria (Alfouzan and Dhar, 2017). In the last few decades, a shift towards high-antifungal resistance with nonalbicans Candida (NAC) infections has been noted (Chandak et al., 2018). 
Biofilms are complex communities of microorganisms formed and adhered to various types of surfaces, which secrete an exopolymeric substance known as a matrix (Rishabh et al., 2017). Candida species have the ability to produce biofilm which protects them from the defense of the host immune system and antifungal drugs, and it is one of the particular characteristics of Candida pathogenicity (Pakshir et al., 2017; Cavalheiro and Teixeira, 2018), and increased their ability to adhesion on biotic and abiotic surfaces (Thamke et al., 2014). In C. albicans, hyphal differentiation is a crucial characteristic in biofilm development, influencing its structure and function, whereas C. glabrata lacks this capacity, being reported to form pseudo-hyphae (Pereira, 2018).

Recently, clinical infections caused by non-albicans Candida (NAC) have been described regularly, and several of these isolates have been shown to produce biofilms (Rishabh et al., 2017). The advantages of forming a biofilm include environmental protection, metabolic cooperation, nutrient availability, and the acquisition of recent genetic characteristics (Mohandas and Ballal, 2011).

Biofilms have an essential function as pathogenic agent reservoirs, allowing coinfection with other pathogens, promoting infection persistence, and increasing mortality rates (Pakshir et al., 2017). The study objectives were to isolate and identify Candida spp. from urine specimens and to detect the ability of Candida isolates to biofilm formation. Also, to compare the biofilm formation between C. albicans and C. glabrata isolates.

\section{MATERIALS AND METHODS}

\section{A. Identification of isolates}

The study was conducted over six months (September 2020 to March 2021), in this study, 174 female diabetic patients were included who attend for diabetic follow up at the AlWafa Specialized Center for Diabetes and Endocrinology, Mosul city, Iraq, with ages ranged from 16 to 72 years and who suffer from UTI symptoms. Diabetic patients who have taken any medications, corticosteroids or immunosuppressive therapy during data collection were excluded.

Firstly, clean-catch midstream urine specimens were collected using a sterile, wide-mouth plastic container. Then, the specimens were brought to the laboratory for processing within two hours.

After that, the standard microbiological techniques were used for culturing the specimens on sabouraud dextrose agar (SDA) at $37^{\circ} \mathrm{C}$ for $24-48$ hours of incubation or longer if required. On the other hand, to confirm all doubtful yeast colonies, traditional techniques were used including gram staining, and the germ tube test and chlamydospore formation on cornmeal agar (Forbes et al., 2007) For differentiation, these isolates sub-cultured on HiCrome agar medium ( HiCrome Candida Differential Agar, HiMedia, India) and incubated at $37^{\circ} \mathrm{C}$ for 48 hours (Helmy, 2012).

\section{B. Biofilm Formation}

To detect biofilm formation, two different techniques were employed, as described below:

\section{Tube method}

The tube method (TM) as described previously by Gokce et al. (2007) was used to determine biofilms forming activity, a loopful of each Candida isolates grew on SDA plate was inoculated into polystyrene tubes with screw caps, each one containing $10 \mathrm{ml}$ of Sabouraud dextrose broth (SDB) with $8 \%$ glucose and inoculated tubes have been incubated at $35^{\circ} \mathrm{C}$ for 48 hours. After incubation, the broth in tubes was aspirated gently and cleaned once with distilled water. After discarding the medium and yeast cells, the tubes were stained with $1 \%$ safranin, after 10 minutes, the stain was decanted. To remove any remaining discolouration, the tubes were washed with distilled water. The presence of the visible adherent film lined the wall and the bottom of tubes indicated biofilm formation.

The test was performed in triplicate and the results were scored as negative - , mild + , moderate ++ , and strong +++ . Two observers read the results independently (Shin et al., 2002).

\section{Congo red agar method}

For screening isolates that produce biofilm in an alternative technique, congo red agar medium (CRA) used and composed of brain heart infusion broth (BHI) $(37 \mathrm{~g} / \mathrm{L})$, sucrose $(50 \mathrm{~g} / \mathrm{L})$, agar $(20 \mathrm{~g} / \mathrm{L})$, and congo red stain (0.8 g/L) (Janakiram et al., 2017). Congo red stain was prepared separately as a concentrated aqueous solution and autoclaved at $121^{\circ} \mathrm{C}$ for 15 minutes prior to addition separately after the agar had cooled to $55^{\circ} \mathrm{C}$. Plates were inoculated and incubated at $37^{\circ} \mathrm{C}$ for $2-3$ days.

According to Janakiram et al. (2017), black colonies with a dry crystalline indicated positive results. The color of a non-biofilm producers colonies is usually pink. The tests were done in triplicate and were repeated three times.

\section{RESULtS}

In our study of the identification method. Out of the total 174 specimens, yeast species were isolated from $56(32.2 \%)$ specimen (Figure 1). Out of the 56 isolates, 50 isolates were positive for Candida species, majority were C. glabrata 30 isolates $(60 \%)$ which were the predominant species and followed by 18 isolates $(36 \%)$ of $C$. albicans and 2 isolates (4\%) of C. krusei (Figure 2). 


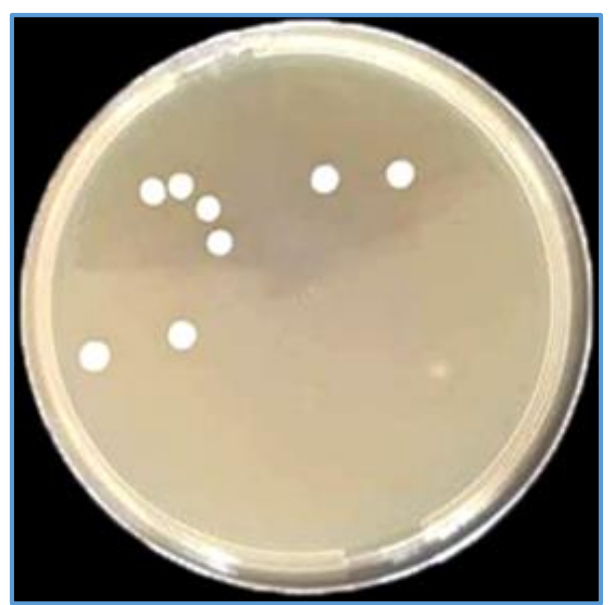

Figure 1: Yeast colonies on SDA plate

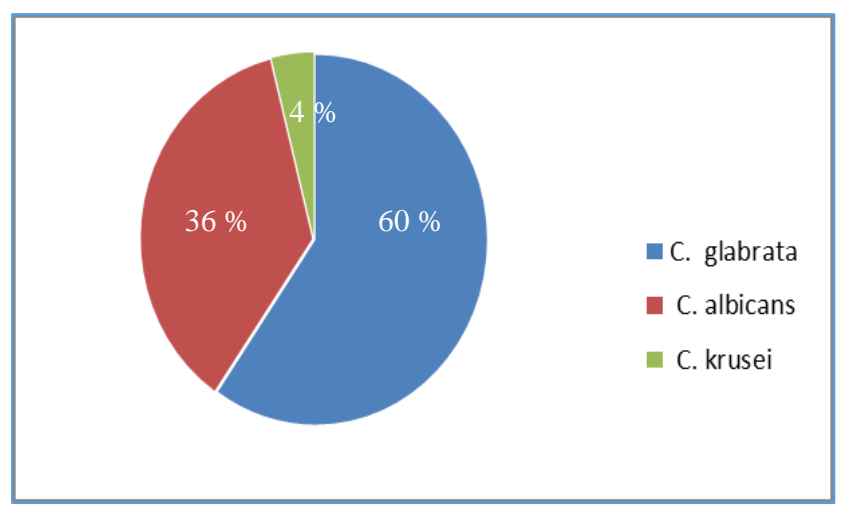

Figure 2: The frequency of Candida species isolated from urine of female diabetic patients.

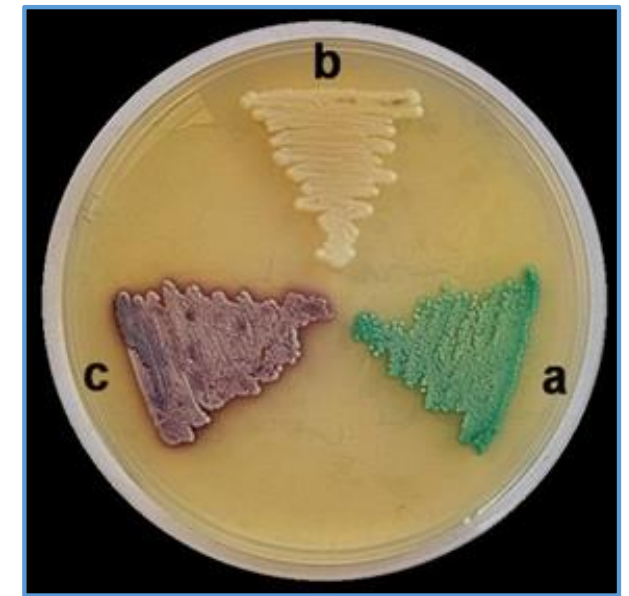

Figure 3: Different Candida species on HiCrome Candida differential agar plate. a: C. albicans, b: C. glabrata, c: C. krusei.

Out of 56 yeast isolates, the 18 isolates (positive to germ tube test and chlamydospore formation test) were grown on HiCrome Candida Differential Agar medium in light green color which indicated to the species C. albicans. The 38 isolates, 30 of them were grown in cream to white and 2 isolates were grown in purple, fuzzy color which indicated to $C$. glabrata and $C$. krusei respectively, but the 6 of isolates were grown in pink color and this color was not defined by manufacturer instructions (Figure 3). C. albicans is detected with a high degree of sensitivity and specificity by this medium, only in rare cases may a $C$. albicans isolate not turn to green color, for this reason, a germ tube test is required for a more certain diagnosis (Reiss et al., 2012).

In tube method test for biofilm production, the results have been noted that the number of non-biofilm producers of $C$. albicans were 2 isolates $(11.1 \%)$, the mild producers were 6 isolates $(33.3 \%)$, the moderate producers were $10(55.6 \%)$, and no strong producers appeared among $C$. albicans isolates.

Whereas in C. glabrata isolates, the results of the number of non-biofilm producers were 1 isolate $(3.3 \%)$, the mild producers were 3 isolates $(10 \%)$, the moderate producers were $20(66.7 \%)$, and 6 isolates $(20 \%)$ were observed as strong producers (Table 1; Figure 4).

Table 1. Biofilm formation among Candida isolates by tube method

\begin{tabular}{|l|c|c|c|c|c|}
\hline \multirow{2}{*}{ Candida spp. } & \multirow{2}{*}{$\begin{array}{c}\text { No. of biofilm } \\
\text { negative isolates }\end{array}$} & \multicolumn{3}{|c|}{$\begin{array}{c}\text { No. of biofilm } \\
\text { positive isolates }\end{array}$} & \multirow{2}{*}{ Total } \\
\cline { 3 - 5 } & & $1+$ & $2+$ & $3+$ & \\
\hline C. albicans & 2 & 6 & 10 & 0 & 18 \\
C. glabrata & 1 & 3 & 20 & 6 & 30 \\
\hline
\end{tabular}

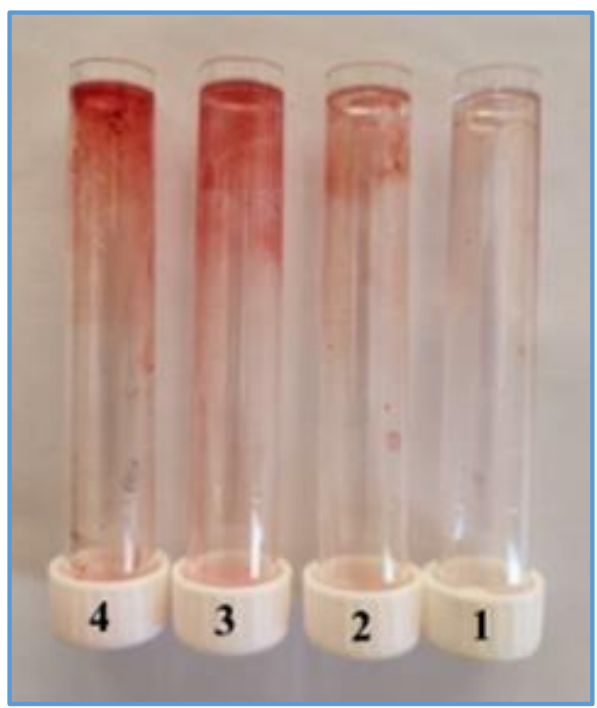

Figure 4: Biofilm formation by tube method 1: Negative, 2: Mild, 3 : Moderate, 4: Strong.

In biofilm formation test on congo red agar plates, the results revealed one morphotype for $C$. albicans isolates were 18 isolates $(100 \%)$ with pink color colonies which indicated nonbiofilm producers and there are no biofilm producers, while in $C$. glabrata isolates, the results showed two different morphotypes, were 27 isolates (90\%) nonproducer with pink color colonies and 3 isolates (10\%) producers were colonies appeared in black color with crystalline appearance (Table 2; Figure 5). 
Table 2. Biofilm formation among Candida isolates by congo red agar method.

\begin{tabular}{|c|c|c|c|}
\hline Candida spp. & $\begin{array}{c}\text { No. of biofilm } \\
\text { negative isolates }\end{array}$ & $\begin{array}{c}\text { No. of biofilm } \\
\text { positive isolates }\end{array}$ & Total \\
\hline C. albicans & 18 & 0 & 18 \\
C. glabrata & 27 & 3 & 30 \\
\hline
\end{tabular}

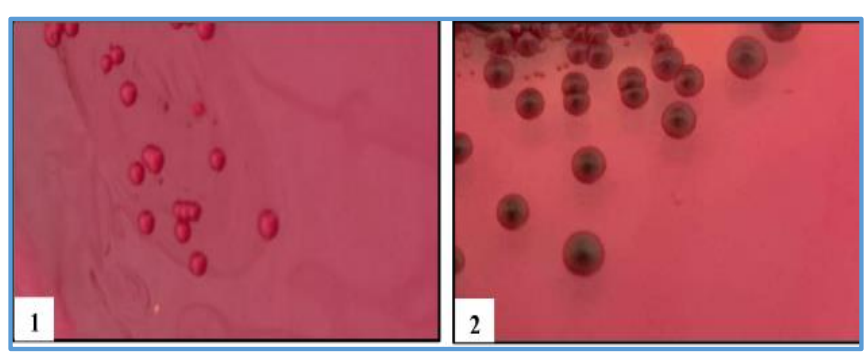

Figure 5: Biofilm formation by tube method 1: Negative isolate, 2: Positive isolate

\section{DISCUSSION}

Our results showed that the majority of Candida species identified in 50 positive isolates were $C$. glabrata which appeared in 30 isolates $(60 \%)$ and followed by $C$. albicans $(36 \%)$. These results were in agreement with another study showed that $C$. glabrata was the most prevalent Candida species etiology of candiduria $(50 \%)$ in diabetic patients, followed by $C$. albicans (31.6\%) and C. krusei $(10.5 \%)$ (Falahati et al., 2016). Furthermore, the study of Zakhem et al. (2021) showed C. glabrata as the predominant species among non-albicans Candida in candidemia cases. Recent findings (Sahai and Kumar, 2018; Pramodhini et al., 2021) have also been detected that non-albicans Candida species were predominant in urinary tract infections. In recent years, we noted that non-albicans Candida species prevalence was more than $C$. albicans. Epidemiological data revealed that non-albicans Candidia infections are rising globally (Taei et al., 2019). Many studies have suggested that the distribution of Candida species that cause candiduria varies regionally (Toner et al., 2016).

In recent years, we noted that non-albicans Candidia species such as $C$. glabrata, the prevalence was more than $C$. albicans which may be ascribed to their potential for adaptation to the conditions of the urinary tract as well as their inherent and/or acquired resistance to conventional antifungals, $C$. glabrata poses a threat to health and a potential cause of therapeutic failure (Falahati et al., 2016; Goyal et al., 2016).

Results of this study showing majority of the clinical isolates of $C$. albicans and $C$. glabrata exhibiting biofilmproducing abilities by TM methods in contrast with CRA were only 3 isolates of $C$. glabrata biofilm producers similar to that of previous studies of Nerurkar et al. (2012) and Chandak et al. (2018) which observed highest biofilm activity in non-albicans Candida species.

A biofilm is a highly structured community of microorganisms that are embedded in a matrix of polysaccharides linked to one another at a surface or interface (Sardi et al., 2014). Biofilm-associated human infections are becoming more common in clinical settings, accounting for $65 \%$ to $80 \%$ of all infections, biofilm has been identified as a key source of chronic human infections in several investigations using modern microscopy and molecular techniques (Pereira, 2018).

Although the majority of studies have focused on $C$. albicans in the biofilm formation subject, several authors have found that other Candida species and other yeast genera, as well as filamentous fungi, are also involved in biofilm development (Sardi et al., 2014).

\section{V.CONCLUSIONS}

Results of our study showed that the majority of $C$. albicans and $C$. glabrata isolates were producers for biofilm by TM method the contrary to CRA methods. As a result, we can conclude that TM is the best conventional method, easy to use and sensitive to detect biofilm-forming yeast whereas the CRA method, is an unreliable method. Furthermore, our results also showed $C$. glabrata is evolving into one of the most prevalent pathogenic yeasts in urinary tract infections of female diabetic patients.

Keeping in mind the high incidence rate of candiduria in people who have diabetes, control of diabetes (especially type 2), risk factors (such as extremes of age and female sex) and causative connections between diabetes and candiduria should all be emphasized.

\section{CONFLICT OF INTEREST}

The authors declare no conflict of interest

\section{REFERENCES}

Alfouzan, W.A., Dhar, R. (2017). Candiduria: evidence-based approach to management, are we there yet?. Journal de mycologie medicale, 27(3), 293-302.

Cavalheiro, M., Teixeira, M.C. (2018). Candida biofilms: threats, challenges, and promising strategies. Frontiers in medicine, 5 , 28.

Chandak, R.J., Mishra, B., Thakur, A., Loomba, P., Dogra, V. (2018) Comparison of methods of biofilm detection in urinary Candida isolates and evaluating its role in persistent candiduria. National Journal of Laboratory Medicine, 7(2), 60-116.

Devi, A.R., Hymavathi, R., Mounika, G. (2019). Candida Species Isolation, Identification and Biofilm Detection at a Tertiary Care Hospital. Int $J$ Cont Med Res, 6(4), 45.

Falahati, M., Farahyar, S., Akhlaghi, L., Mahmoudi, S., Sabzian, K., Yarahmadi, M., Aslani, R. (2016). Characterization and identification of candiduria due to Candida species in diabetic patients. Current medical mycology, 2(3), 10.

Forbes, B.A., Sahm, D.F., Weissfeld, A.S. (2007). Bailey and Scott's Diagnostic Microbiology. $12^{\text {th }}$ ed., Mosby, Elsevier, Inc., U.S.A, pp. $710-711$.

Geerlings, S., Fonseca, V., Castro-Diaz, D., List, J., Parikh, S. (2014). Genital and urinary tract infections in diabetes: impact of pharmacologically-induced glucosuria. Diabetes research and clinical practice, 103(3), 373-381.

Gokce, G., Cerikcioglu, N., Yagci, A. (2007). Acid proteinase, phospholipase, and biofilm production of Candida species isolated from blood cultures. Mycopathologia, 164(6), 265-269.

Goyal, R. K., Sami, H., Mishra, V., Bareja, R., Behara, R.N. (2016). Nonalbicans candiduria: an emerging threat. J Appl Pharm Sci, 6(3), 48-50.

He, Z., Huo, X., Lei, D., Zhao, H., Jia, K., Wang, F. (2021). Management of candiduria in hospitalized patients: a single-center study on 
the implementation of IDSA guidelines and factors affecting clinical decisions. European Journal of Clinical Microbiology \& Infectious Diseases, 40(1), 59-65.

Helmy, M.M. (2012). Phenotypic analysis of Candida Species Associated with Vulvovaginal Candidiasis. Egypt J Med Microbiol, 21(1), 109-115.

Janakiram, B., Myneni, R.B., Kumar, K.A., Gousia, Sk., Latha, J.N.L. (2017). Methods of Determination of Biofilm Formation by Candida albicans. Res. J. Microbiol., 12(1): 90-96.

Mohandas, V., Ballal, M. (2011). Distribution of Candida species in different clinical samples and their virulence: biofilm formation, proteinase and phospholipase production: a study on hospitalized patients in southern India. Journal of Global Infectious Diseases, 3(1), 4.

Nerurkar, A., Solanky, P., Chavda, N., Baria, H., Desai, B. (2012). Isolation of Candida Species in clinical specimens and its virulence factor: The biofilm. International Journal of Medical Science and Public Health, 1 (2), 97-100.

Pakshir, K., Bordbar, M., Zomorodian, K., Nouraei, H., Khodadadi, H. (2017). Evaluation of CAMP-like effect, biofilm formation, and discrimination of Candida africana from vaginal Candida albicans species. Journal of pathogens, 2017.

Pereira, D.G. (2018). Biofilm formation in Candida glabrata: the role of the Transcription Factor Tec1. M.Sc. thesis, Tecnico School, Lisboa, Portugal.

Pieralli, F., Bazzini, C., Vannucchi, V., Mancini, A., Nozzoli, C. (2014). A case of Candida glabrata severe urinary sepsis successfully treated with micafungin. Medical mycology case reports, 5, 1-3.

Pramodhini, S., Srirangaraj, S., Easow, J.M. (2021). Candiduria-Study of Virulence Factors and Its Antifungal Susceptibility Pattern in Tertiary Care Hospital. Journal of Laboratory Physicians, 13(3), 231-237.

Reiss, E, Shadomy, H.J., Lyon, G.M. (2012). Fundamental Medical Mycology. John Wiley and Sons, Inc., USA. p. 44.

Rishabh, V., Jain Pavithra, K.R., Kulkarni, R.D. (2017). Pathogenicity Testing of Clinical Candida Isolates by Assessing Biofilm Formation and their Adhesion to Urinary Catheter Material. Journal of Pure and Applied Microbiology, 11(4), 1939-1946.

Sahai, S., Kumar, A. (2018). Role of Candida in Catheter Associated Urinary Tract Infection. Int J Cur Res Rev, 10(20), 15-9.

Sardi, J.D.C.O., Pitangui, N.D.S., Rodríguez-Arellanes, G., Taylor, M.L., Fusco-Almeida, A.M., Mendes-Giannini, M.J.S. (2014). Highlights in pathogenic fungal biofilms. Revista iberoamericana de micologia, 31(1), 22-29.

Shin, J.H., Kee, S.J., Shin, M.G., Kim, S.H., Shin, D.H., Lee, S. K., Ryang, D.W. (2002). Biofilm production by isolates of Candida species recovered from nonneutropenic patients: comparison of bloodstream isolates with isolates from other sources. Journal of Clinical Microbiology, 40(4), 1244-1248.

Taei, M., Chadeganipour, M., Mohammadi, R. (2019). An alarming rise of non-albicans Candida species and uncommon yeasts in the clinical samples; a combination of various molecular techniques for identification of etiologic agents. BMC research notes, 12(1), 1-7.

Thamke, D.C., Tayade, H.A., Tayade, S.A. (2014). Biofilm formation among Candida albicans isolated from vagina. IJBAR, 20, 0512.

Toner, L., Papa, N., Aliyu, S.H., Dev, H., Lawrentschuk, N., Al-Hayek, S. (2016). Candida growth in urine cultures: a contemporary analysis of species and antifungal susceptibility profiles. QJM: An International Journal of Medicine, 109(5), 325-329.

Woldemariam, H.K., Geleta, D.A., Tulu, K.D., Aber, N.A., Legese, M.H., Fenta, G.M., Ali, I. (2019). Common uropathogens and their antibiotic susceptibility pattern among diabetic patients. BMC infectious diseases, 19(1), 1-10.

Zakhem, A.E., Istambouli, R., Alkozah, M., Gharamti, A., Tfaily, M.A., Jabbour, J.F., Araj, G.F., Tamim, H., Kanj, S.S. (2021). Predominance of Candida glabrata among non-albicans Candida species in a 16-year study of candidemia at a tertiary care center in Lebanon. Pathogens, 10(1), 82. 